\title{
Single-Source AIGaAs Frequency Comb Transmitter for 661 Tbit/s Data Transmission in a 30-core Fiber
}

Hu, Hao; Da Ros, Francesco; Ye, Feihong; Pu, Minhao; Ingerslev, Kasper; Porto da Silva, Edson; Nooruzzaman, Md; Amma, Yoshimichi; Sasaki, Yusuke; Mizuno, Takayuki

Total number of authors:

19

Published in:

CLEO: Applications and Technology 2016

Link to article, DOI:

10.1364/CLEO_AT.2016.JTh4C.1

Publication date:

2016

Document Version

Peer reviewed version

Link back to DTU Orbit

Citation (APA):

Hu, H., Da Ros, F., Ye, F., Pu, M., Ingerslev, K., Porto da Silva, E., Nooruzzaman, M., Amma, Y., Sasaki, Y., Mizuno, T., Miyamoto, Y., Ottaviano, L., Semenova, E., Guan, P., Zibar, D., Galili, M., Yvind, K., Oxenløwe, L. K., \& Morioka, T. (2016). Single-Source AIGaAs Frequency Comb Transmitter for 661 Tbit/s Data Transmission in a 30-core Fiber. In CLEO: Applications and Technology 2016 Optical Society of America (OSA). https://doi.org/10.1364/CLEO_AT.2016.JTh4C.1

\section{General rights}

Copyright and moral rights for the publications made accessible in the public portal are retained by the authors and/or other copyright owners and it is a condition of accessing publications that users recognise and abide by the legal requirements associated with these rights.

- Users may download and print one copy of any publication from the public portal for the purpose of private study or research.

- You may not further distribute the material or use it for any profit-making activity or commercial gain

- You may freely distribute the URL identifying the publication in the public portal 


\title{
Single-Source AlGaAs Frequency Comb Transmitter for 661 Tbit/s Data Transmission in a 30-core Fiber
}

\author{
Hao Hu${ }^{1}$, Francesco Da Ros ${ }^{1}$, Feihong Ye ${ }^{1}$, Minhao Pu${ }^{1}$, Kasper Ingerslev ${ }^{1}$, Edson Porto da Silva ${ }^{1}$, \\ Md. Nooruzzaman ${ }^{1}$, Yoshimichi Amma ${ }^{2}$, Yusuke Sasaki ${ }^{2}$, Takayuki Mizuno ${ }^{3}$, Yutaka Miyamoto ${ }^{3}$, \\ Luisa Ottaviano ${ }^{1}$, Elizaveta Semenova ${ }^{1}$, Pengyu Guan ${ }^{1}$, Darko Zibar ${ }^{1}$, Michael Galili ${ }^{1}$, Kresten Yvind ${ }^{1}$, \\ Leif K. Oxenløwe ${ }^{1}$, and Toshio Morioka ${ }^{1}$

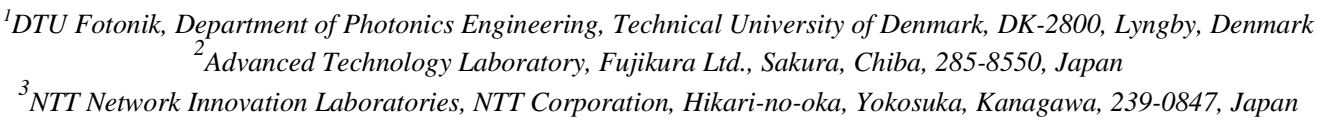

\begin{abstract}
We demonstrate an AlGaAs-on-insulator nano-waveguide-based frequency comb with high OSNR enabling a single-source to fully load a 9.6-km heterogeneous 30-core fibre with $661 \mathrm{Tbit} / \mathrm{s}$ data achieved by 30xcores, 80xWDM, 40 Gbaud, and PDM-16QAM.

OCIS codes: (060.2330) Fiber optics communications; (060.4230) Multiplexing.
\end{abstract}

\section{Introduction}

With the introduction of space-division multiplexing (SDM), the world has seen an explosion in reported records of data transmission throughput, such as the accomplishment of crossing the $1 \mathrm{Pbit} / \mathrm{s}$ border in 2012 [1-2]. As cost and energy consumption are becoming limiting factors in high-capacity systems, using fewer lasers with less energy consumption grows desirable and frequency comb based single source transmission has attracted great research interest [3-6]. In this paper, we present the first photonic-chip based frequency comb, relying on spectral broadening of a mode-locked laser comb in an AlGaAs-on-insulator (AlGaAsOI) nano-waveguide, with a sufficient comb output power to support several hundred Tbit/s of optical data. The high comb OSNR allows us to send the 80 WDM PDM channels over 30 spatial channels, and we demonstrate successful $9.6 \mathrm{~km}$ transmission in a heterogeneous 30-core fiber reaching a total capacity of $661 \mathrm{Tbit} / \mathrm{s}$.

\section{SPM based frequency comb generation in an AIGaAsOI nano-waveguide}

The AlGaAsOI nano-waveguide (Fig. 1(a)) has recently emerged as an ultra-efficient nonlinear medium, since it combines high intrinsic material nonlinearity (on the order of $10^{-17} \mathrm{~W} / \mathrm{m}^{2}$ ), a high-index contrast as silicon-oninsulator, and low linear loss [7]. In addition, the bandgap of AlGaAs can be engineered by changing the $\mathrm{Al}$ concentration to avoid two-photon absorption (TPA) at telecom wavelengths. All of this renders AlGaAsOI nanowaveguide a good source candidate for self-phase modulation (SPM) based optical frequency comb generation.

\section{Experimental setup and results}

The experimental setup is shown in Fig. 1 (c). The single source laser in the transmitter is an Erbium glass oscillating modelocked laser, which produces $10-\mathrm{GHz}$ pulses (1542 nm, 1.5-ps FWHM), with a spectrum as shown in Fig. 1 (d). The pulses are amplified and used to generate an optical frequency comb based on SPM in the AlGaAsOI photonic chip (Fig. 1 (b)), with an average launched power of $19.3 \mathrm{dBm}$ (peak power of $\sim 5.6 \mathrm{~W}$ ). Fig. 1(e) shows the broadened spectrum at the output of the AlGaAsOI nano-waveguide, which has a 20-dB bandwidth of $\sim 44 \mathrm{~nm}$.

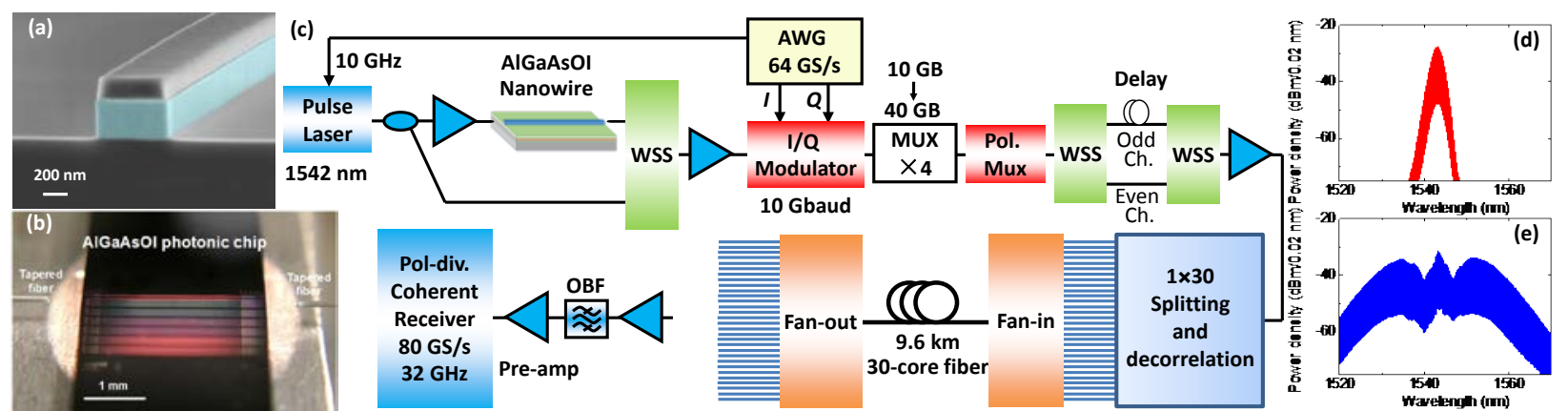

Fig. 1. (a) SEM picture of a fabricated AlGaAsOI nano-waveguide (denoted by the artificial blue color). (b) Photograph of the AlGaAsOI photonic chip. (c) Schematic of the experimental setup for the $661 \mathrm{Tbit} / \mathrm{s}$ single-source AlGaAs frequency comb transmitter for 30 -core transmission demonstration. (d) Input mode locked laser pulse spectrum to AlGaAs nano-waveguide, and (e) the corresponding output frequency comb spectrum. 
(a)

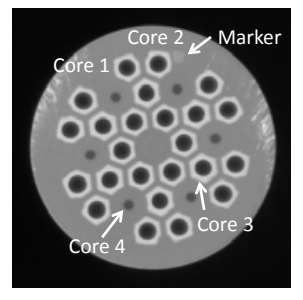

(b)

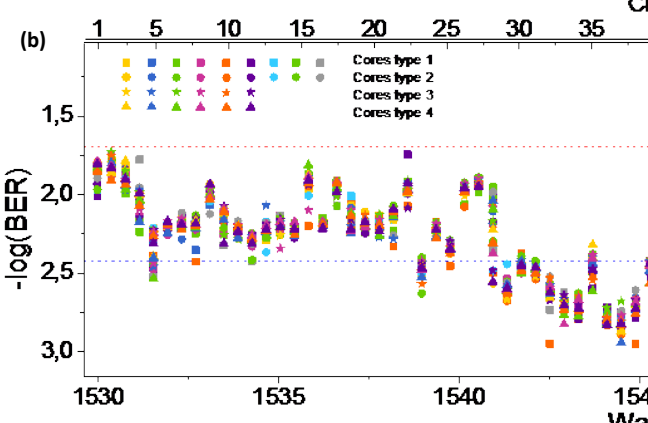

Channel (\#)
40

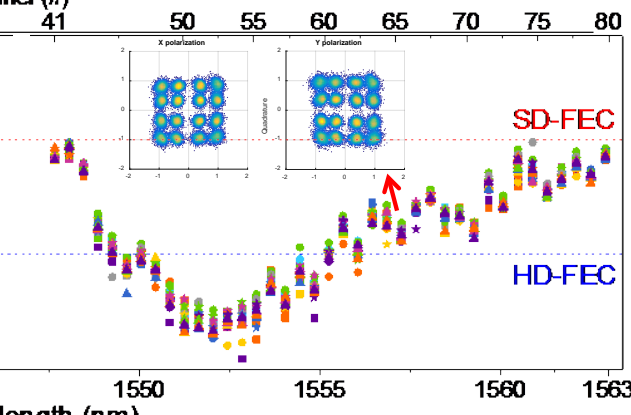

Fig. 2. (a) Cross-sectional view of the 30-core fiber; (b) BERs of all the 30 spatial channels for 80 measured wavelengths between 1529.97 nm and $1562.92 \mathrm{~nm}$. 80 wavelengths have the BERs below SD-FEC limit of which 22 wavelengths have the BERs even below HD-FEC limit for all the 30 SDM sub-channels. Inset: constellation diagram of the $66^{\text {th }}$ WDM channel after the transmission.

The estimated OSNR is $\sim 43 \mathrm{~dB}$ at $1552 \mathrm{~nm}$ and $\sim 30 \mathrm{~dB}$ at $1563 \mathrm{~nm}$. The broadened spectrum is equalized in a wavelength selective switch (WSS) and the inhomogeneous part (from $1540.73 \mathrm{~nm}$ to $1545.87 \mathrm{~nm}$ ) in the center is replaced by the original spectrum from the mode-locked laser through another path, which results in a flat and stable frequency comb [3]. The generated frequency comb with $10 \mathrm{GHz}$ spacing is modulated with 10 Gbaud 16QAM in a standard IQ modulator driven by a 60 Gsample/s arbitrary waveform generator (AWG). The modulated 10 Gbaud 16QAM signal is multiplexed in time to 40 Gbaud using a passive fiber-delay multiplexer (MUX $\times 4$ ) and then polarization multiplexed to the resulting $320 \mathrm{Gbit} / \mathrm{s}$ PDM-16QAM signal.

To generate a WDM signal with $50 \mathrm{GHz}$ spacing, the broadened spectrum is spectrally sliced into odd and even channels and separated into two paths using a second WSS. The delay difference between the two paths is 7.5 ns in order to de-correlate the odd and even channels. The WSS is programmed for rectangular filtering with a bandwidth of $40 \mathrm{GHz}$ and $50 \mathrm{GHz}$ spacing, in order to generate 40 Gbaud Nyquist-OTDM PDM-16QAM signals for all the WDM carriers. The odd and even WDM channels are recombined using a third WSS. 30 de-correlated SDM channels are then generated using splitters, amplifiers and delays (at least $2.5 \mathrm{~ns}$ between SDM channels) and then launched into the 9.6-km 30-core single-mode fiber through a 3D-waveguide based fan-in device. The launched power for each core is between 14-18 dBm accounting for loss-variations in the fan-in/fan-out from $5 \mathrm{~dB}$ to $8 \mathrm{~dB}$.

The heterogeneous 30-core fiber has four different types of cores, as shown in Fig. 2 (a), to realize the highdensity core arrangement with low cross-talk (XT) [8]. The 30-core fiber has $\mathrm{A}_{\mathrm{eff}}$ of $\sim 80 \mu \mathrm{m}^{2}$ and low XT of below $50 \mathrm{~dB}$ at $9.6 \mathrm{~km}$. The 30 cores are arranged within the limited cladding diameter of $228 \mu \mathrm{m}$.

At the output of the 30-core fiber, the 30 spatial channels are demultiplexed using another 3D-waveguide based fan-out device. A tunable bandpass filter with a bandwidth of $50 \mathrm{GHz}$ is used to select each WDM channel. The selected WDM channels are detected with a coherent receiver followed by a digital sampling oscilloscope.

Fig. 2 (b) shows the BERs of all the 30 spatial channels for the 80 measured wavelengths between $1529.97 \mathrm{~nm}$ and $1562.92 \mathrm{~nm}$ (for each WDM/SDM channel, BERs of 2 polarizations and 4 OTDM tributaries are averaged). All channels are present in all cores simultaneously ensuring the validity of the claimed transmission throughput. 80 WDM channels are below FEC limits, 58 below SD-FEC (20\% overhead) and 22 below HD-FEC (7\% overhead), yielding a line rate of $768 \mathrm{Tbit} / \mathrm{s}$ and a net rate after FEC overhead subtraction of $661 \mathrm{Tbit} / \mathrm{s}$.

\section{Conclusions}

We have demonstrated the first photonic chip based single-source transmitter based on an AlGaAsOI nanowaveguide frequency comb capable of carrying $661 \mathrm{Tbit} / \mathrm{s}$ data in a fully loaded 30-core fiber. This is the highest reported amount of data carried on the light generated from a chip-based single-source transmitter, and we demonstrate successful $9.6 \mathrm{~km}$ transmission. We employ 16QAM modulation, PDM and WDM of 40 Gbaud channels in addition to 30xSDM and all 2400 PDM-16QAM channels are simultaneously generated, transmitted and individually measured to be below the FEC threshold after transmission.

\section{Acknowledgements}

SPOC research centre of excellence, ref DNRF123, NATEC Villum centre of excellence and EU-Japan coordinated R\&D project on "Scalable And Flexible optical Architecture for Reconfigurable Infrastructure (SAFARI)" commissioned by the MIC, Japan and EC Horizon 2020.

\section{References}

[1] H. Takara et al., ECOC 2012, paper Th.3.C.1 (2012).

[2] D. Qian et al., FiO 2012, paper FW6C.3 (2012)

[3] D. Hillerkuss et al, NPHOT, 5, 364-371 (2011)

[4] V. Ataie et al, OFC 2014, postdeadline paper Th5B.7 (2014)).
[5] H. Hu et al, CLEO 2014 postdeadline paper JTh5B.10 (2014)

[6] B. Puttnam et al, ECOC 2015, paper PDP.3.1 (2015).

[7] M. Pu et al, OFC 2015, postdeadline paper Th5A.3 (2015).

[8] Y. Amma et al, OFC 2015, paper Th4C.4 (2015). 